\title{
A Characterization of Markovian Homogeneous Multicomponent Gaussian Fields
}

\author{
G. O. S. Ekhaguere \\ Department of Mathematics, University of Ibadan, Ibadan, Nigeria
}

\begin{abstract}
Necessary and sufficient conditions are given for a certain class of homogeneous multicomponent Gaussian generalized stochastic fields to possess a Markov property equivalent to Nelson's. The class of Markov fields so characterized has as a subclass the class of Markov fields which lead by Nelson's Reconstruction Theorem to some covariant (free) quantum fields.
\end{abstract}

\section{Introduction}

In 1948, Levy [1] defined a Brownian motion indexed by the $d$-dimensional Euclidean space $\mathbb{R}^{d}$. With this Brownian motion in mind, he introduced [2] the notion of Markov property of finite order for (ordinary) stochastic fields, and conjectured [2] that the Brownian motion indexed by $\mathbb{R}^{2 v+1}, v=a$ nonnegative integer, is Markovian of order $v+1$. It was left to McKean [3] to establish the veracity of Levy's conjecture. Recently, Molchan [4] has furnished an alternative proof of Levy's conjecture while Pitt [5] has extended McKean's proof to arbitrary Gaussian stochastic fields which are Markovian of some finite order. In his proof, Molchan employs aspects of the theory of elliptic partial differential equations and the notion of the reproducing kernel Hilbert space [6] associated with a stochastic field. By a blending of the preceding ideas with those of McKean [3] and by applying Peetre's characterization [7] of differential operators, Pitt characterized a finite order Markovian Gaussian stochastic field, under some assumptions, by identifying the inner product of its reproducing kernel Hilbert space with the Dirichlet form [8] of a strongly elliptic partial differential operator.

Results, of the above type are, of course, certainly of relevance at the initial stages of development of a theory of Markov stochastic fields. But stochastic fields which are Markovian of finite order are clearly only a special class of Markovian fields. Indeed, McKean employs a generalization of Levy's notion of Markov property in [3] and his extended definition thus accommodates Markov stochastic fields which are not necessarily of finite order. In [9] and [10], necessary and sufficient conditions for a homogeneous scalar Gaussian stochastic field to be 
Markovian in McKean's sense are proffered under some assumptions on the spectral measure of the stochastic field.

In this paper, we consider a class of multicomponent Gaussian generalized stochastic fields subjected to minimal covariance constraints and we study a notion of Markov property, which implies and is implied by Nelson's [11] notion of Markov property, for this class of fields. Our results generalize the results of [9] and [10]. In Constructive Quantum Field Theory, Nelson has developed a scheme $[11,12]$ for generating quantum fields from a certain class of Markov fields which have more restrictive covariance properties than we impose here. Given the central role now being played by Markov fields in contemporary Quantum Field Theory $[13,14]$, a characterization of the above mentioned quantum-field-yielding class, or even a wider class such as we study here, of Markov fields is evidently desirable. Such a characterization immediately also leads to a characterization of the associated class of quantum fields (by Nelson's Reconstruction Theorem [11]). On the other hand, a study of this nature is clearly also of independent interest in abstract Probability Theory.

\section{Ultradistributions and a Paley-Wiener Theorem}

In this section, we undertake a cursory review of some of the mathematical structures employed in the sequel.

We begin with the ultradifferentiable functions. In describing the space of ultradifferentiable functions, one assumes given a fixed infinite sequence

$$
\mathbf{M}=\left(M_{k}\right)_{0 \leqq k \leqq \infty}
$$

of positive numbers distinguished by the following properties:

$$
\begin{aligned}
& M_{k}^{2} \leqq M_{k-1} M_{k+1}, \text { for } k \geqq 1 ; \\
& \sum_{k=1}^{\infty} \frac{M_{k-1}}{M_{k}}<+\infty ;
\end{aligned}
$$

(1.3) there are constants $A$ and $H$ such that

$$
M_{k+1} \leqq A H^{k} M_{k}, \quad k \geqq 0 ;
$$

(1.4) there is a constant $C$ such that

$$
\left(\frac{k !}{j !(k-j) !}\right) M_{k-j} M_{j} \leqq C M_{k}, \quad \text { for all } k \text { and } j \text { with } \quad 0 \leqq j \leqq k .
$$

(1.5) Remark. The need for the conditions (1.1) through (1.4) on the infinite sequence $\mathbf{M}=\left(M_{k}\right)_{0 \leqq k \leqq \infty}$ of positive numbers will transpire below.

Let $M$ be defined on $(0, \infty)$ as follows :

$$
M(\lambda)=\log \left[\sup _{k \in \mathbb{N}}\left(\frac{\lambda^{k} M_{0}}{M_{k}}\right)\right], \quad \lambda \in(0, \infty),
$$

where $\mathbb{N}$ is the set of all natural numbers. 
Then (1.2) is equivalent [15] to

$(1.2)^{* *} \int_{0}^{\infty} d \lambda \frac{M(\lambda)}{\lambda^{2}}<\infty$.

We shall be employing the equivalence of (1.2) and (1.2)** in the sequel; in this connection, wherever the function $M$ appears below, it shall be assumed, without further comments, to be defined by $(1.2)^{*}$ and to satisfy (1.2)**.

Hereafter, $U$ denotes an arbitrary, non-void open subset of $\mathbb{R}^{d}, d \in \mathbb{N}$, and we shall write $\operatorname{Comp}(U)$ for the collection of all compact convex subsets of $U$. Furthermore, $C_{0}^{\infty}(U)$ will denote the linear space of all complex-valued $C^{\infty}$ functions with compact support in $U$. In the sequel, we shall be working with the Cartesian product $\operatorname{Comp}(U) \times(0, \infty)$. In this connection, if $(K, l)$ and $\left(K^{\prime}, l^{\prime}\right)$ belong to $\operatorname{Comp}(U) \times(0, \infty)$, we shall write

$$
(K, l) \leqq\left(K^{\prime}, l^{\prime}\right) \text { whenever } K \subset K^{\prime}, l \leqq l^{\prime} .
$$

The fundamental function spaces which are employed below will now be introduced.

(1.7) Definition. The Space $\mathscr{D}^{\mathbf{M}}(U)$

Let $(K, l)$ belong to $\operatorname{Comp}(U) \times(0, \infty)$. Then $\mathscr{D}^{\mathbf{M}}(U, K, l)$ will denote the set of all functions $f$ in $C_{0}^{\infty}(U)$, with support in $K$, such that

$$
\begin{aligned}
& \|f\|_{U, K, l}=\sup _{\substack{x \in k \in K \\
|x|=k, k \in \mathbb{N}}}\left[\frac{\left|\left(D^{\alpha} f\right)(x)\right|}{l^{k} M_{k}}\right]<+\infty, \\
& D^{\alpha}=\frac{\partial^{|\alpha|}}{\partial x^{\alpha_{1}} \partial x^{\alpha_{2}} \ldots \partial x^{\alpha_{d}}}, \quad|\alpha|=\alpha_{1}+\alpha_{2}+\ldots+\alpha_{d} .
\end{aligned}
$$

We note that with the norm given by $(1.8), \mathscr{D}^{\mathbf{M}}(U, K, l)$ is in fact a Banach space. Furthermore, for $(K, l)$ and $\left(K^{\prime}, l^{\prime}\right)$ belonging to $\operatorname{Comp}(U) \times(0, \infty)$, with $(K, l) \leqq\left(K^{\prime}, l^{\prime}\right)$, we have

$$
\mathscr{D}^{\mathbf{M}}(U, K, l) \subset \mathscr{D}^{\mathbf{M}}\left(U, K^{\prime}, l^{\prime}\right),
$$

and the injection of $\mathscr{D}^{\mathbf{M}}(U, K, l)$ into $\mathscr{D}^{\mathbf{M}}\left(U, K^{\prime}, l^{\prime}\right)$ is compact [15].

We denote by $\mathscr{D}^{\mathbf{M}}(U)$ the inductive limit

$$
\mathscr{D}^{\mathbf{M}}(U)=\operatorname{ind}_{\substack{K \rightarrow U \\ l \rightarrow+\infty}} \mathscr{D}^{\mathbf{M}}(U, K, l)
$$

of $\mathscr{D}^{\mathbf{M}}(U, K, l)$ as $K$ and $l$ increase monotonically to $U$ and $+\infty$, respectively.

The Space $\mathscr{D}^{\mathbf{M}}(K, l)$. This is the Banach space of all functions $f$ in $C^{\infty}\left(\mathbb{R}^{d}\right)$ with support in $K \subset \mathbb{R}^{d}$ such that

$$
\|f\|_{k, l}=\sup _{\substack{x \in K \\|\alpha|=k, k \in \mathbb{N}}}\left[\frac{\left|\left(D^{\alpha} f\right)(x)\right|}{l^{k} M_{k}}\right]<+\infty .
$$


We remark that $\mathscr{D}^{\mathbf{M}}(K, l) \subset \mathscr{D}^{\mathbf{M}}\left(K, l^{\prime}\right)$ whenever $l<l^{\prime}$, and the imbedding of $\mathscr{D}^{\mathbf{M}}(K, l)$ in $\mathscr{D}^{\mathbf{M}}\left(K, l^{\prime}\right)$ is compact. Denote by $\mathscr{D}^{\mathbf{M}}(K), K$ compact and convex, the following inductive limit

$$
\mathscr{D}^{\mathbf{M}}(K)=\operatorname{ind}_{l \rightarrow+\infty} \lim _{\mathscr{D}^{\mathbf{M}}}(K, l)
$$

of $\mathscr{D}^{\mathbf{M}}(K, l)$ as $l$ increases monotonically to $+\infty$.

The Space $\mathscr{E}^{\mathbf{M}}(U)$. Finally, we define $\mathscr{E}^{\mathbf{M}}(U)$ as the space of all functions $f$ in $C^{\infty}(U)$ such that for every $K \in \operatorname{Comp}(U)$, the restriction $\left.f\right|_{K}$ of $f$ to $K$ belongs to $\mathscr{D}^{\mathbf{M}}(K)$.

From the above definitions, it is clear that the spaces $\mathscr{D}^{\mathbf{M}}(U), \mathscr{D}^{\mathbf{M}}(K, l)$, and $\mathscr{E}^{\mathbf{M}}(U)$ are all nuclear.

\section{(1.10) Remark}

(i) We call $\mathscr{D}^{\mathbf{M}}(U)$ the space of ultradifferentiable functions on $U$ of Roumieu type $[16,17]$, so as to distinguish it from the space $\mathscr{B}^{\mathbf{M}}(U)$ of ultradifferentiable functions on $U$ of Beurling type [18], which we do not define here. However, all the results we obtain below remain valid when $\mathscr{B}^{\mathbf{M}}(U)$ is substituted for $\mathscr{D}^{\mathbf{M}}(U)$.

(ii) The space $\mathscr{D}^{\mathbf{M}}(U)$ has the following attributes:

(a) it admits an approximate identity, i.e. for every sphere $S(\varepsilon)$ of radius $\varepsilon>0$ contained in $U$, there exists an $f(\cdot, \varepsilon)$ in $\mathscr{D}^{\mathbf{M}}(U)$ with the properties:

$$
\operatorname{supp} f(\cdot, \varepsilon) \subset S(\varepsilon), f(x, \varepsilon)>0 \quad \text { for all } \quad x \in S(\varepsilon),
$$

and $\int_{U} d x f(x, \varepsilon)=1, \varepsilon>0$;

(b) for every open covering of $U$, there is a partition of unity by elements of $\mathscr{D}^{\mathbf{M}}(U)$;

(c) differentiation is a bounded operator on $\mathscr{D}^{\mathbf{M}}(U)$;

(d) $\mathscr{D}^{\mathbf{M}}(U)$ is an algebra under pointwise multiplication;

(e) $\mathscr{D}^{\mathbf{M}}(U)$ is a dense subset of the Schwartz space $\mathscr{D}(U)$ of compex-valued $C^{\infty}$ functions with compact support in $U$.

We note that properties (a), (b), and (e) follow from (1.1) and (1.2) while (c) follows from (1.3) and (d) from (1.4). This remark underlines the need for the conditions (1.1) through (1.4).

(iii) Under the operation of pointwise multiplication, $\mathscr{D}^{\mathbf{M}}(U)$ is an ideal of $\mathscr{E}^{\mathbf{M}}(U)$

(1.11) Definition. The space of ultradistributions of Roumieu type, with support contained in $U$, is defined as the dual $\mathscr{D}^{\prime \mathbf{M}}(U)$, with strong dual topology, of $\mathscr{D}^{\mathbf{M}}(U)$.

(1.12) Remark. Let $\langle\cdot, \cdot\rangle$ denote the duality pairing of elements of $\mathscr{D}^{\prime \mathbf{M}}(U)$ and $\mathscr{D}^{\mathbf{M}}(U)$. The operations of differentiation in $\mathscr{D}^{\mathbf{M}}(U)$ and pointwise multiplication of elements of $\mathscr{D}^{\mathbf{M}}(U)$ by functions in $\mathscr{E}^{\mathbf{M}}(U)$ are defined thus: with $F \in \mathscr{D}^{\mathbf{M}}(U)$ and $g \in \mathscr{E}^{\mathbf{M}}(U)$, then $g F$ and $D^{\alpha} F$ are the ultradistributions which satisfy

(i) $\langle g F, f\rangle=\langle f, g f\rangle$,

(ii) $\left\langle D^{\alpha} F, f\right\rangle=(-1)^{|\alpha|}\left\langle F, D^{\alpha} f\right\rangle$, for all $f \in \mathscr{D}^{\mathbf{M}}(U)$. 


\section{(1.13) Remark}

(i) In the above review, we have followed closely the survey in Lions and Magenes [19].

(ii) The next result, which generalizes Theorem 4 in [15], gives a characterization of $\mathscr{D}^{\mathbf{M}}(U)$ by means of its Fourier-Laplace image.

(1.14) A Paley-Wiener Theorem. An ultradistribution $F$ belongs to $\mathscr{D}^{\mathbf{M}}(U)$ if and only if there are positive numbers $b$ and $c$ such that the Fourier-Laplace transform

$$
z \mapsto \tilde{F}(z)=\int d x e^{-i x \cdot z} F(x)
$$

of $F$ satisfies

$$
|\tilde{F}(z)| \leqq c \exp \left(-M\left(\frac{|z|}{b}\right)+H_{U}(z)\right)
$$

where

$$
\begin{aligned}
& H_{U}(z)=\sup _{x \in U} \operatorname{Im}(x \cdot z) \\
& x \cdot z=x_{1} z_{1}+x_{2} z_{2}+\ldots+x_{d} z_{d},\left(x_{j}, z_{j}\right) \in \mathbb{R} \times \mathbb{C}, \quad j=1,2, \ldots, d
\end{aligned}
$$

( $H_{U}$ is called the support function of the open set $U$ ).

(1.17) Remark

(i) The open set $U$ occuring in the preceding theorem may be replaced with any convex set $K$ without prejudice to its validity.

(ii) Let $U$ be an open subset of $\mathbb{R}^{d}$. In the sequel, we shall have cause to employ certain pseudo-differential operators. The general definition of the latter is as follows :

Let $P(\cdot, \cdot)$ be any member of $C^{\infty}\left(U \times \mathbb{R}^{d}\right)$ with the property that there are real numbers $m, \varrho$, and $\delta$, with $\varrho>0$ and $\delta \geqq 0$, such that for every compact subset $K \subset U$ and for all $\alpha=\left(\alpha_{1}, \ldots, \alpha_{d}\right), \beta=\left(\beta_{1}, \ldots, \beta_{d}\right)$ in $\mathbb{N}^{d}$, we have

$$
\left|D_{x}^{\beta} D_{\xi}^{\alpha} P(x, \xi)\right| \leqq C_{\alpha, \beta, K}(1+|\xi|)^{m-\varrho|\alpha|+\delta|\beta|}
$$

for all $(x, \xi) \in K \times \mathbb{R}^{d}$, where $C_{\alpha, \beta, K}$ is some positive constant and $|\alpha|=\alpha_{1}+\ldots+\left|\alpha_{d}\right|$. Then $P(\cdot, \cdot)$ is called a symbol and the integral

$$
(2 \pi)^{-d} \int d \xi P(x, \xi) \tilde{u}(\xi) e^{i x \cdot \xi}
$$

is absolutely convergent for every $u \in C_{0}^{\infty}(U)$, where $\tilde{u}$ here denotes the Fourier transform of $u$. The operator $P(x, D), x \in K$, which maps $C_{0}^{\infty}(U)$ into $C^{\infty}(U)$, defined as follows:

$$
P(x, D) u(x)=(2 \pi)^{-d} \int d \xi P(x, \xi) \tilde{u}(\xi) e^{i x \cdot \xi}, u \in C_{0}^{\infty}(U),
$$

is called a pseudo-differential operator of order $m$ and type $(\varrho, \delta)$.

We remark that the pseudo-differential operators employed by us below do not depend on $x$, i.e. for such pseudo-differential operators, the symbol $(x, \xi) \mapsto P(x, \xi)$ is independent of $x$.

(iii) To us, Theorem (1.14) is an important result which we employ below. It leads rapidly to the following equally important result which may also be found in [15]. 
(1.18) Theorem. Let

(1.19) $z \mapsto J(z)=\sum_{|\alpha|=0}^{\infty} a_{\alpha} z^{\alpha}$

$$
\begin{aligned}
& z^{\alpha}=z_{1}^{\alpha_{1}} z_{2}^{\alpha_{2}} \ldots z_{d}^{\alpha_{d}}, z_{j} \in \mathbb{C}, \quad j=1,2, \ldots, d \\
& a_{\alpha}=\text { complex constants, } \alpha \in \mathbb{N}^{d},
\end{aligned}
$$

be an entire function which satisfies the growth condition that for any $b>0$, there is $a$ positive number $c>0$ such that

(1.20) $|J(z)| \leqq c \exp (M(b|z|)), \quad z \in \mathbb{C}^{d}$.

Then for any open subset $U$ of $\mathbb{R}^{d}$, the pseudo-differential operator

(1.21) $J(D)=\sum_{|\alpha|=0}^{\infty} a_{\alpha} D^{\alpha}$,

which is rigorously defined as in Remark (1.17) (ii), maps $\mathscr{D}^{\mathbf{M}}(U)$ continuously into itself. Furthermore, the sequence

$$
S_{n}(f)=\sum_{|\alpha|=0}^{n} a_{\alpha} D^{\alpha} f, \quad n=0,1,2, \ldots
$$

of partial sums is absolutely convergent in the topology of $\mathscr{D}^{\mathbf{M}}(U)$ for any $f$ in $\mathscr{D}^{\mathbf{M}}(U)$.

(1.22) Definition. An entire function $J$ which satisfies inequality (1.20) will be called a multiplier for the Fourier-Laplace image of $\mathscr{D}^{\mathbf{M}}(U)$.

(1.23) Remark

(i) Obviously, if $J$ is a multiplier for the Fourier-Laplace image of $\mathscr{D}^{\mathbf{M}}(U)$, then $J(D)$ is a local operator on $\mathscr{D}^{\mathbf{M}}(U)$, i.e. $\mathscr{D}^{\mathbf{M}}(U)$ is invariant under $J(D)$ :

$$
J(D) \mathscr{D}^{\mathbf{M}}(U) \subseteq \mathscr{D}^{\mathbf{M}}(U) .
$$

(ii) We note here that the spaces $\left\{\mathscr{D}^{\mathbf{M}}(U): U\right.$ is open in $\mathbb{R}^{d}, \mathbf{M}=\left(M_{k}\right)_{0 \leqq k \leqq \infty}$ fixed satisfy the next property:

if $U_{1}$ and $U_{2}$ are open subsets of $\mathbb{R}^{d}$ such that

$$
U_{2} \supseteqq U_{1} \text {, then }
$$

$$
\mathscr{D}^{\mathbf{M}}\left(U_{1}\right) \cong \mathscr{D}^{\mathbf{M}}\left(U_{2}\right)
$$

and this imbedding is compact.

(1.24) Definition

(i) We denote by $\mathscr{D}^{\mathbf{M}}\left(\mathbb{R}^{d}\right)$ the following inductive limit

$$
\mathscr{D}^{\mathbf{M}}\left(\mathbb{R}^{d}\right)=\text { ind } \lim _{U \rightarrow \mathbb{R}^{d}} \mathscr{D}^{\mathbf{M}}(U)
$$

of $\mathscr{D}^{\mathbf{M}}(U)$ as $U$ increases monotocially to $\mathbb{R}^{d}$.

(ii) The strong dual of $\mathscr{D}^{\mathbf{M}}\left(\mathbb{R}^{d}\right)$ will be denoted by $\mathscr{D}^{\prime \mathbf{M}}\left(\mathbb{R}^{d}\right)$.

(1.25) Remark. With the foregoing preliminaries, we may now introduce the class of generalized stochastic fields considered in this paper. 


\section{Homogeneous Multicomponent Generalized Stochastic Fields and Markov Property}

Let $(\Omega, \mathscr{B}, \mu)$ be a complete probability space. We denote by $L^{0}(\Omega, \mathscr{B}, \mu)$ the space of all complex-valued random variables on $(\Omega, \mathscr{B}, \mu)$; we make $L^{0}(\Omega, \mathscr{B}, \mu)$ into a topological space by endowing it with the topology of convergence in measure.

(2.1) Definition. A continuous linear map from $\mathscr{D}^{\mathbf{M}}\left(\mathbb{R}^{d}\right)$ into the topological space $L^{0}(\Omega, \mathscr{B}, \mu)$ is called a generalized random variable on $(\Omega, \mathscr{B}, \mu)$. And, by an $N$ component generalized stochastic field on $(\Omega, \mathscr{B}, \mu)$, we mean a collection

$$
H^{0}(\Omega)=\left\{\xi(f)=\left(\xi_{j}(f): j=1,2, \ldots, N\right): f \in \mathscr{D}^{\mathbf{M}}\left(\mathbb{R}^{d}\right)\right\}
$$

of $N$-tuples of generalized random variables on $(\Omega, \mathscr{B}, \mu)$.

(2.2) Remark

(i) We assume throughout this paper that $H^{0}(\Omega)$ is of second order i.e.

$$
\xi(f) \in H^{0}(\Omega) \quad \text { implies } \quad \xi_{j}(f) \in L^{2}(\Omega, \mathscr{B}, \mu), f \in \mathscr{D}^{\mathbf{M}}\left(\mathbb{R}^{d}\right), \quad j=1,2, \ldots, N .
$$

Let $\mathbb{M}(f)$ and $B\left(f^{(2)}, f^{(1)}\right)$ denote the matrices whose entries are

$$
\mathbb{M}_{j}(f)=\left\langle 1, \xi_{j}(f)\right\rangle_{L^{2}(\Omega, \mathscr{B}, \mu)}, f \in \mathscr{D}^{\mathbf{M}}\left(\mathbb{R}^{d}\right),
$$

and

$$
B_{i j}\left(f^{(2)}, f^{(1)}\right)=\left\langle\xi_{i}\left(f^{(2)}\right), \xi_{j}\left(f^{(1)}\right)\right\rangle_{L^{2}(\Omega, \mathscr{B}, \mu)}, f^{(k)} \in \mathscr{D}^{\mathbf{M}}\left(\mathbb{R}^{d}\right),
$$

$k=1,2$, respectively, where $\langle\cdot, \cdot\rangle_{L^{2}(\Omega, \mathscr{B}, \mu)}$ is the inner product of $L^{2}(\Omega, \mathscr{B}, \mu)$. It is clear that $\mathbb{I}(f)$ is the column vector of expectation functionals of components of $\xi(f) \in H^{0}(\Omega)$ while $B\left(f^{(2)}, f^{(1)}\right)$ is the matrix of their correlation functionals.

(ii) Throughout the rest of this paper, we assume that $H^{0}(\Omega)$ is Gaussian, i.e. finite collections of members of $H^{0}(\Omega)$ have Gaussian probability distribution.

(iii) In addition to the assumption in (ii), we are interested in this paper only in $N$-component generalized stochastic fields which have the transformation property mentioned in the next definition.

(2.3) Definition. Let $\alpha \mapsto \tau_{\alpha}$ be the representation of $\mathbb{R}^{d}$ in $\operatorname{Aut}\left(\mathscr{D}^{\mathbf{M}}\left(\mathbb{R}^{d}\right)\right)$ defined as follows :

$$
\left(\tau_{\alpha} f\right)(x)=f(x-\alpha), x \in \mathbb{R}^{d} .
$$

Then $H^{0}(\Omega)$ is called a homogeneous $N$-component generalized stochastic field if it is such that the column vector $\mathbb{M}(f)$ of expectation functionals and the matrix $B\left(f^{(2)}, f^{(1)}\right)$ of correlation functionals of an arbitrary member $\xi(f)=\left(\xi_{j}(f): j=1,2, \ldots, N\right), f \in \mathscr{D}^{\mathbf{M}}\left(\mathbb{R}^{d}\right)$, in $H^{0}(\Omega)$ satisfy

(i) $\mathbb{M}\left(\tau_{\alpha} f\right)=\mathbb{M}(f)$,

(ii) $B\left(\tau_{\alpha} f^{(2)}, \tau_{\alpha} f^{(1)}\right)=B\left(f^{(2)}, f^{(1)}\right)$

for all $\alpha \in \mathbb{R}^{d}$ and all $f, f^{(1)}, f^{(2)}$ in $\mathscr{D}^{\mathbf{M}}\left(\mathbb{R}^{d}\right)$.

(2.4) Remark. In connection with homogeneous $N$-component generalized stochastic fields, there is the following result. 
(2.5) Theorem. The $N$-component generalized stochastic field $H^{0}(\Omega)$ is homogeneous if and only if the matrices $\mathbb{M}(f)$ and $B\left(f^{(2)}, f^{(1)}\right)$ have the following representations :

(i) $\mathbb{M}(f)=\mathrm{m} \int d x f(x)$,

(ii) $B\left(f^{(2)}, f^{(1)}\right)=\int \mathbb{F}(d p) \overline{\tilde{f}}^{(2)}(p) \tilde{f}^{(1)}(p)$

for all $f, f^{(1)}, f^{(2)}$ in $\mathscr{D}^{\mathbf{M}}\left(\mathbb{R}^{d}\right)$, where: $\mathbf{m}$ is an arbitrary constant $N$-component column vector, and

$\Lambda \mapsto \mathbb{F}(\Lambda)=\left(\mathbb{F}_{i j}(\Lambda): i, j=1,2, \ldots, N\right)$,

$\Lambda=$ Borel subset of $\mathbb{R}^{d}$, is a positive-definite matrix of complex measures, on the $\sigma$ algebra generated by the Borel subsets of $\mathbb{R}^{d}$, which satisfy the following condition:

$$
\int\left|\mathbb{F}_{i j}(d p)\right| \exp \left(-M(\phi(|p|))+H_{K}(p)\right)<\infty,
$$

for all compact convex subsets $K$ of $\mathbb{R}^{d}$ and all increasing functions $\phi$ such that

$$
\lim _{\lambda \rightarrow \infty} \frac{\phi(\lambda)}{\lambda}=0 .
$$

(2.6) Remark. Since the proof of Theorem (2.5) is straight-forward, we shall omit it.

There is no loss of generality in assuming that the arbitrary constant column vector occurring in (2.5)(i) is indeed the zero column vector. Hence, in what follows, we set $\mathrm{m}=0$, the zero column vector.

Additionally, we shall assume in the sequel that the complex measures $\Lambda \mapsto \mathbb{F}_{i j}(\Lambda), i, j=1,2, \ldots, N$, are given by complex-valued densities $p \mapsto \mathbb{F}_{i j}(p)$, i.e.

(2.7) $\quad \mathbb{F}_{i j}(\Lambda)=\int_{\Lambda} d p \mathbb{F}_{i j}(p), \Lambda=$ Borel subset of $\mathbb{R}^{d}$,

which satisfy:

$\operatorname{det} \mathbb{F}(p) \equiv \operatorname{det}\left(\mathbb{F}_{i j}(p)\right) \neq 0, \quad$ for almost all $p$ in $\mathbb{R}^{d}$,

with respect to $d$-dimensional Lebesgue measure.

For convenience, we list below all the assumptions we have imposed thus for on $H^{0}(\Omega)$ :

(i) $H^{0}(\Omega)$ is of second order and is Gaussian;

(ii) $H^{0}(\Omega)$ is a mean zero, homogeneous $N$-component generalized stochastic field;

(iii) the matrix $\Lambda \mapsto \mathbb{F}(\Lambda)$ of spectral measures of members of $H^{0}(\Omega)$ has entries which may be represented as in (2.7); furthermore, the matrix $\left.p \mapsto \mathbb{F}(p)=\mathbb{F}_{i j}(p)\right)$ of densities occurring in (2.7) is pointwise invertible for almost every $p \in \mathbb{R}^{d}$, with respect to $d$-dimensional Lebesgue measure.

We shall not waive any of the assumptions in (2.8) throughout the discussion. (2.9) Remark. To conclude this section, we introduce next the notion of Markov 
property studied by us in the following section. In this connection, let $\mathscr{D}^{\mathbf{M}, N}\left(\mathbb{R}^{d}\right)$ denote the $N$-fold Cartesian product of $\mathscr{D}^{\mathbf{M}}\left(\mathbb{R}^{d}\right)$ with itself, and set

$$
\mathbb{B}\left(\mathbf{f}^{(2)}, \mathbf{f}^{(1)}\right)=\sum_{i, j=1}^{N} \int d p \overline{\tilde{f}}_{i}^{(2)}(p) \mathbb{F}_{i j}(p) \tilde{f}_{j}^{(1)}(p),
$$

for $\mathbf{f}^{(k)} \in \mathscr{D}^{\mathbf{M}, N}\left(\mathbb{R}^{d}\right), k=1,2$.

Clearly, $\mathbb{B}(\cdot, \cdot)$ is a continuous Hermitean functional on $\mathscr{D}^{\mathbf{M}, N}\left(\mathbb{R}^{d}\right) \times \mathscr{D}^{\mathbf{M}, N}\left(\mathbb{R}^{d}\right)$ which is antilinear in its first argument and linear in the second argument. Furthermore, in view of the positive-definiteness of the matrix

$$
\mathbb{F}(\Lambda)=\left(\mathbb{F}_{i j}(\Lambda): i, j=1,2, \ldots, N\right),
$$

for every Borel $\Lambda \subset \mathbb{R}^{d}$, it follows that $\mathbb{B}(\mathbf{f}, \mathbf{f}) \geqq 0$, for all $\mathbf{f} \in \mathscr{D}^{\mathbf{M}, N}\left(\mathbb{R}^{d}\right)$, i.e. the functional $\mathbb{B}(\cdot, \cdot)$ is itself positive-definite. This fact enables us to make the next definition.

(2.11) Definition. Let $\mathscr{H}\left(\mathbb{R}^{d}\right)$ denote the uniquely determined Hilbert space of $N$-component vectors which possesses $\mathbb{B}(\cdot, \cdot)$ as its reproducing functional (for this notion, which generalizes the notion of a reproducing kernel introduced by Aronszajn [6], see [20], for example), and let $\langle\cdot, \cdot\rangle_{\mathscr{H}\left(\mathbb{R}^{d}\right)}$ denote the inner product of $\mathscr{H}\left(\mathbb{R}^{d}\right)$.

(2.12) Remark. We note the following properties which connect the reproducing functional $\mathbb{B}(\cdot, \cdot)$ of $\mathscr{H}\left(\mathbb{R}^{d}\right)$ with the inner product $\langle\cdot, \cdot\rangle_{\mathscr{H}\left(\mathbb{R}^{d}\right)}$ of $\mathscr{H}\left(\mathbb{R}^{d}\right)$ :

(i) for each $\mathbf{g} \in \mathscr{D}^{\mathbf{M}, N}\left(\mathbb{R}^{d}\right)$, the vector-valued ultradistribution

$\mathbb{B}_{\mathbf{g}}(\cdot): \mathscr{D}^{\mathbf{M}, N}\left(\mathbb{R}^{d}\right) \mapsto \mathbb{C}, \quad$ the complex numbers

$$
\mathbf{f} \mapsto \mathbb{B}_{\mathbf{g}}(\mathbf{f})=\mathbb{B}(\mathbf{g}, \mathbf{f})
$$

belongs to $\mathscr{H}\left(\mathbb{R}^{d}\right)$;

(ii) the Hilbert space $\mathscr{H}\left(\mathbb{R}^{d}\right)$ is generated by

$\left\{\mathbb{B}_{\mathbf{g}}(\cdot): \mathbf{g} \in \mathscr{D}^{\mathbf{M}, N}\left(\mathbb{R}^{d}\right)\right\} ;$

(iii) for all $(\mathbf{f}, \mathbf{g}) \in \mathscr{D}^{\mathbf{M}, N}\left(\mathbb{R}^{d}\right) \times \mathscr{D}^{\mathbf{M}, N}\left(\mathbb{R}^{d}\right)$, there is the equality:

$\left\langle\mathbb{B}_{\mathbf{f}}, \mathbb{B}_{\mathbf{g}}\right\rangle_{\mathscr{H}\left(\mathbb{R}^{d}\right)}=\mathbb{B}(\mathbf{f}, \mathbf{g})$.

In the sequel, we employ the foregoing properties without further comments.

(2.13) Definition. Let $U$ be any open subset of $\mathbb{R}^{d}$ and $K$ be any closed subset or an arbitrary convex subset of $\mathbb{R}^{d}$. Then, we denote by $\mathscr{H}(U)$ the sub-Hilbert space of $\mathscr{H}\left(\mathbb{R}^{d}\right)$ generated by

$$
\left\{\mathbb{B}_{\mathbf{f}}(\cdot): \mathbf{f} \in \mathscr{D}^{\mathbf{M}, N}\left(\mathbb{R}^{d}\right), \operatorname{supp} \mathbf{f} \subseteq U\right\},
$$

and we define $\mathscr{H}(K)$ by

$$
\mathscr{H}(K)=\bigcap_{\mathcal{O} \supset \mathrm{K}} \mathscr{H}(\mathcal{O})
$$

where the intersection is taken over all open subsets $\mathcal{O}$ of $\mathbb{R}^{d}$ which contain $K$. 
In the sequel, $P_{K}$ will denote the projection of $\mathscr{H}\left(\mathbb{R}^{d}\right)$ onto $\mathscr{H}(K)$, where $K$ is an open, a closed or an arbitrary convex subset of $\mathbb{R}^{d}$.

The Hilbert space $\mathscr{H}\left(\mathbb{R}^{d}\right)$ is isometrically isomorphic to the Hilbert space $H\left(\mathbb{R}^{d}\right)$ which is the completion of $\mathscr{D}^{\mathbf{M}, N}\left(\mathbb{R}^{d}\right)$ in the topology afforded by the norm derived from the inner product

$$
(\mathbf{f}, \mathbf{g}) \mapsto\langle\mathbf{f}, \mathbf{g}\rangle_{H\left(\mathbb{R}^{d}\right)}=\mathbb{B}(\mathbf{f}, \mathbf{g}), \mathbf{f}, \mathbf{g} \in \mathscr{D}^{\mathbf{M}, N,\left(\mathbb{R}^{d}\right) .}
$$

From property (i) of (2.12), it follows that each $\mathbf{h}$ in $\mathscr{H}\left(\mathbb{R}^{d}\right)$ has a representation of the form

(2.14a) $\mathbf{h}=\mathbb{F}^{*}(D) \mathbf{f}, \quad$ for some $\quad \mathbf{f} \in H\left(\mathbb{R}^{d}\right)$,

where $\mathbb{F}^{*}(p)$ denotes the matrix which is adjoint (i.e. the transposed complex conjugate) to $\mathbb{F}(p), p \in \mathbb{R}^{d}$, and $p \mapsto \mathbb{F}_{i j}(p)$ are the spectral functions occurring in (2.7).

For any open $U$ in $\mathbb{R}^{d}, H(U)$ is the sub-Hilbert space of $H\left(\mathbb{R}^{d}\right)$ generated by $\left\{\mathbf{f} \in \mathscr{D}^{\mathbf{M}, N}\left(\mathbb{R}^{d}\right)\right.$ : supp $\left.\mathfrak{f} \subseteq U\right\}$; for $K$ closed or convex, the sub-Hilbert space $H(K)$ is now defined as for $\mathscr{H}(K)$. We note too that $H(K)$ and $\mathscr{H}(K)$ are isometrically isometric, with each $\mathbf{h}$ in $\mathscr{H}(K)$ admitting a representation of the form

(2.14b) $\mathbf{h}=\mathbb{F}^{*}(D) \mathbf{f}, \quad$ for some $\mathbf{f} \in H(K)$.

From (iii) above, we deduce that if $\mathbf{u}, \mathbf{v}$ belong to $\mathscr{H}\left(\mathbb{R}^{d}\right)$, then

$$
\langle\mathbf{u}, \mathbf{v}\rangle_{\mathscr{H}\left(\mathbb{R}^{d}\right)}=\int d p \tilde{\mathbf{u}}^{*}(p) \mathbb{F}^{*-1}(p) \tilde{\mathbf{v}}(p),
$$

where $\tilde{\mathbf{u}}^{*}$ is the transposed conjugate of the column vector $\tilde{\mathbf{u}}$. Since $\langle\cdot, \cdot\rangle_{\mathscr{H}\left(\mathbb{R}^{d}\right)}$ is an inner product, and hence separately continuous in each of its two arguments, and by the nuclearity of $\mathscr{H}\left(\mathbb{R}^{d}\right)$, it follows from the Abstract Kernel Theorem ([21], p. 73-79) that the inverse Fourier transforms of the entries of the matrix $p \mapsto \mathbb{F}^{-1}(p), p \in \mathbb{R}^{d}$ are (translation-invariant) ultradistributions on $\mathscr{D}^{\mathbf{M}}\left(\mathbb{R}^{d} \times \mathbb{R}^{d}\right)$, which are clearly not identically zero.

We make use of (2.14) and (2.15) below.

It is now opportune to introduce the notion of Markow property discussed in the next section.

(2.16) Definition. Let $U$ be an arbitrary open subset of $\mathbb{R}^{d}$ with complement $U^{\prime}$, closure $\bar{U}$ and boundary $\partial U$. Then

$$
H^{0}(\Omega)=\left\{\xi(f)=\left(\xi_{j}(f): j=1,2, \ldots, N\right): f \in \mathscr{D}^{\mathbf{M}}\left(\mathbb{R}^{d}\right)\right\}
$$

is said to have the Markov property if and only if

$$
P_{U^{\prime}} P_{\bar{U}}=P_{\partial U} P_{\bar{U}}
$$

or, equivalently, if and only if

$$
P_{U^{\prime}} P_{\bar{U}}=P_{\partial U}
$$

(2.19) Remark.

(i) It is fashionable [11] to formulate the notion of Markov property in terms of $\sigma$-algebras of fields and conditional expectations rather than in terms of Hilbert spaces of vector-valued ultradistributions and orthogonal projections, as we do 
here. Since we deal only with Gaussian fields throughout this paper, the two formulations are equivalent.

(ii) In the next section, we give a complete characterization of fields which have the Markov property.

\section{Necessary and Sufficient Conditions for Markovicity}

We shall now state and prove the main result of this communication. In this regard, we need the following notation.

Let $\mathbb{P}=\left(\mathbb{P}_{i j}\right)$ denote the $N \times N$ matrix whose entries $\mathbb{P}_{i j}$ belong to $\mathscr{D}^{\mathbf{M}}\left(\mathbb{R}^{d}\right)$ and are related to the entries $\left(\mathbb{F}^{-1}\right)_{i j}(\cdot)$ of the matrix $\mathbb{F}^{-1}(\cdot)$ whose adjoint occurs in Eq. (2.15) as follows:

$$
\left\langle\mathbb{P}_{i j}, \tilde{f}\right\rangle=\left\langle\left(\mathbb{F}^{-1}\right)_{i j}, f\right\rangle, \quad \text { for every } \quad f \in \mathscr{D}^{\mathbf{M}}\left(\mathbb{R}^{d}\right),
$$

where $\langle\cdot, \cdot\rangle$ is the duality pairing of members of $\mathscr{D}^{\mathbf{M}}\left(\mathbb{R}^{d}\right)$ and $\mathscr{D}^{\mathbf{M}}\left(\mathbb{R}^{d}\right)$. Thus $\mathbb{P}$ is the matrix of inverse Fourier transforms of the entries of the matrix $\mathbb{F}^{-1}(\cdot)$, which itself exists pointwise almost everywhere in $\mathbb{R}^{d}$ (with respect to $d$-dimensional Lebesgue measure).

Equation (3.1) gives the definition of the ultradistributional Fourier transform $\tilde{\mathbb{P}}=\left(\widetilde{\mathbb{P}}_{i j}\right)$ of the matrix $\mathbb{P}=\left(\mathbb{P}_{i j}\right)$ of ultradistributions and it says simply that

$$
\tilde{\mathbb{P}}(p)=\mathbb{F}^{-1}(p), \quad \text { for almost every } \quad p \in \mathbb{R}^{d} .
$$

We employ (3.2) in the formulation and proof of our theorem, which we now give.

(3.3) Theorem. Let $(\Omega, \mathscr{B}, \mu)$ be a complete probability space and let $H^{0}(\Omega)$ $=\left\{\xi(f)=\left(\xi_{j}(f): j=1,2, \ldots, N\right): f \in \mathscr{D}^{\mathbf{M}}\left(\mathbb{R}^{d}\right)\right\}$ be an $N$-component generalized stochastic field over $(\Omega, \mathscr{B}, \mu)$ which satisfies all the assumptions of (2.8). Then a necessary and sufficient condition for $H^{0}(\Omega)$ to have the Markov property is that each of the functions

$$
p \mapsto \tilde{\mathbb{P}}_{i j}(p), \quad i, j=1,2, \ldots, N, \quad p \in \mathbb{R}^{d},
$$

is, almost everywhere (with respect to d-dimensional Lebesgue measure), the restriction to $\mathbb{R}^{d}$ of an entire function which satisfies an estimate of the form (1.20).

Proof. The condition of the theorem is necessary. For, if $H^{0}(\Omega)$ is indeed Markovian, then

$$
P_{U^{\prime}} P_{\bar{U}}=P_{\partial U}, \quad \text { for every open subset } \mathrm{U} \text { of } \mathbb{R}^{d} \text {. }
$$

Hence, $P_{U}$ f belongs to $\mathscr{H}(\partial U)$ for every $\mathbf{f}$ belonging to $\mathscr{H}(\bar{U})$. This implies that

$$
\left\langle\mathbf{g}, P_{U^{\prime}} \mathbf{f}\right\rangle_{\mathscr{H}\left(\mathbb{R}^{d}\right)}=0
$$

for every $\mathbf{g}$ in $\mathscr{H}\left(U^{\prime 0}\right)$ and for all $\mathbf{f}$ in $\mathscr{H}(\bar{U})$, where $U^{\prime 0}$ denotes the interior of $U^{\prime}$. Since $P_{U} \mathbf{g}=\mathbf{g}$, for every $\mathbf{g} \in \mathscr{H}\left(U^{\prime 0}\right)$, then using Eq. (2.14), Eq. (3.4) becomes :

$$
0=\langle\mathbf{g}, \mathbf{f}\rangle_{\mathscr{H}\left(\mathbb{R}^{d}\right)}=\int d x \mathbf{g}^{*}(x)\left(\mathbb{P}^{*} * \mathbf{f}\right)(x)
$$


for every $\mathbf{g} \in \mathscr{H}\left(U^{\prime 0}\right)$ and very $\mathbf{f} \in \mathscr{H}(\bar{U})$, where $\mathbb{P}^{*} * \mathbf{f}$ denotes the convolution of $\mathbb{P}^{*}$ and $\mathbf{f}$. In particular Eq. (3.5) remains valid if

$$
\mathbf{f}=\left(\begin{array}{c}
0 \\
0 \\
\vdots \\
0 \\
f_{j} \\
0 \\
\vdots \\
0
\end{array}\right), f_{j} \in \mathscr{D}^{\mathbf{M}}(U), \quad j \text { arbitrary }
$$

and

$$
\mathbf{g}=\left(\begin{array}{c}
0 \\
0 \\
\vdots \\
0 \\
g_{k} \\
0 \\
\vdots \\
0
\end{array}\right), g_{k} \in \mathscr{D}^{\mathbf{M}}\left(U^{\prime 0}\right), \quad k \text { arbitrary, }
$$

where the non-vanishing components of $\mathbf{f}$ and $\mathbf{g}$ are $f_{j}$ and $g_{k}$, respectively. Then (3.5) takes the form:

(3.6) $0=\int d x \bar{g}_{k}(x)\left(\overline{\mathbb{P}}_{j k} * f_{j}\right)(x)$.

We shall suppose in the following that the open set $U$ is an arbitrary neighbourhood of the origin of $\mathbb{R}^{d}$.

Let $S(\varepsilon) \subset U$ be a sphere of radius $\varepsilon>0$, centred at the origin of $\mathbb{R}^{d}$, and let $f(\cdot, e), \operatorname{supp} f(\cdot, \varepsilon) \subset S(\varepsilon)$, be an approximate identity for $\mathscr{D}^{\mathbf{M}}\left(\mathbb{R}^{d}\right)$, and set

$$
f_{j}=f(\cdot, \varepsilon), \quad \varepsilon>0
$$

in Eq. (3.6). Then we have

(3.7) $0=\int d x \bar{g}_{j k}(x)\left(\overline{\mathbb{P}}_{j k} * f(\cdot, \varepsilon)\right)(x)$.

In the limit as $\varepsilon$ tends to zero, Eq. (3.7) now yields

$$
0=\int d x g_{k}(x) \mathbb{P}_{j k}(x)
$$

Next, since

(i) $g_{k}$ is an arbitrary member of $\mathscr{D}^{\mathbf{M}}\left(U^{\prime 0}\right)$,

(ii) the open set $U$ is an arbitrary neighbourhood of the origin of $\mathbb{R}^{d}$ which may be made as small as we please, and

(iii) $\mathbb{P}_{j k} \in \mathscr{D}^{\mathbf{M}}\left(\mathbb{R}^{d}\right), j, k=1,2, \ldots, N$, is not the identically zero ultradistribution, it follows from Eq. (3.8) that

(3.9) $\quad \operatorname{supp} \mathbb{P}_{j k}=\{0\}, \quad$ for all $j, k=1,2, \ldots, N$. 
But the support function of the singleton $\{0\}$ is the function which is identically zero. Hence, by Theorem (1.14), $\tilde{\mathbb{P}}_{k j}, k, j=1,2, \ldots, N$, is almost everywhere (with respect to $d$-dimensional Lebesgue measure) the restriction to $\mathbb{R}^{d}$ of an entire function which satisfies an estimate of the form (1.20), where the function $t \rightarrow M(t)$, $t \in(0, \infty)$, occurring in $(1.20)$ is defined by $(1.2)^{*}$ and satisfies $(1.2)^{* *}$. Hence the condition of the theorem is indeed necessary.

The condition of the theorem also suffices. To see this, we need to establish that, under the condition of the theorem, for every open subset $U$ of $\mathbb{R}^{d}$, the vector (3.10) $\mathbf{h}=P_{U^{\prime}} \mathbf{f}$ belongs to $\mathscr{H}(\partial U)$ for every $\mathbf{f}$ in $\mathscr{H}(\bar{U})$.

Now, since $\mathbf{f}$ belongs to $\mathscr{H}(\bar{U})$, there is a $\boldsymbol{\phi}$ in $H(\bar{U})$ such that

$$
\mathbf{f}=\mathbb{F}^{*}(D) \boldsymbol{\phi}, \quad \text { by }(2.14 \mathrm{~b}) \text {. }
$$

Similarly, since $\mathbf{h}$ belongs to $\mathscr{H}\left(\mathbb{R}^{d}\right)$, there is a $\psi$ in $H\left(\mathbb{R}^{d}\right)$ such that

$$
\mathbf{h}=\mathbb{F}^{*}(D) \psi, \quad \text { by }(2.14 \mathrm{a}) \text {. }
$$

The onus is now to prove that $\psi$ does in fact belong to $H(\partial U)$, under the condition of the theorem.

From (3.10), we have

$$
\langle\mathbf{g}, \mathbf{h}\rangle_{\mathscr{H}\left(\mathbb{R}^{d}\right)}=\left\langle\mathbf{g}, P_{U^{\prime}} \mathbf{f}\right\rangle_{\mathscr{H}\left(\mathbb{R}^{d}\right)}=\langle\mathbf{g}, \mathbf{f}\rangle_{\mathscr{H}\left(\mathbb{R}^{d}\right)}
$$

for every $\mathrm{g}$ belonging to $\mathscr{H}\left(U^{\prime 0}\right)$, where $U^{\prime 0}$ is the interior of $U^{\prime}$. By (3.11) and (3.12), Eq. (3.13) becomes:

$$
\left\langle\mathbb{F}^{*}(D) \boldsymbol{\eta}, \mathbb{F}^{*}(D) \psi\right\rangle_{\mathscr{H}\left(\mathbb{R}^{d}\right)}=\left\langle\mathbb{F}^{*}(D) \boldsymbol{\eta}, \mathbb{F}^{*}(D) \boldsymbol{\phi}\right\rangle_{\mathscr{H}\left(\mathbb{R}^{d}\right)},
$$

for every $\mathbf{g} \in \mathscr{H}\left(U^{\prime 0}\right)$ with $\left.\mathbf{g}=\mathbb{F}^{*} D\right) \boldsymbol{\eta}$, for some $\boldsymbol{\eta} \in H\left(U^{\prime 0}\right)$. In particular, Eq. (3.14) holds for every $\mathbf{g} \in \mathscr{H}\left(U^{\prime 0}\right)$ with $\mathbf{g}=\mathbb{F}^{*}(D) \boldsymbol{\eta}$, for some $\boldsymbol{\eta} \in \mathscr{D}^{\mathbf{M}, N}\left(U^{\prime 0}\right)$. Thus, by the isometric isomorphism of $\mathscr{H}\left(\mathbb{R}^{d}\right)$ and $H\left(\mathbb{R}^{d}\right)$, we have from (3.14) that

$$
\begin{aligned}
\langle\boldsymbol{\eta}, \boldsymbol{\psi}\rangle_{\boldsymbol{H}\left(\mathbb{R}^{d}\right)} & =\int d x \boldsymbol{\eta}^{*}(x)(\mathbb{F}(D) \boldsymbol{\psi})(x) \\
& =\langle\boldsymbol{\eta}, \boldsymbol{\phi}\rangle_{\boldsymbol{H}\left(\mathbb{R}^{d}\right)} \\
& =\int d x \boldsymbol{\eta}^{*}(x)(\mathbb{F}(D) \boldsymbol{\phi})(x),
\end{aligned}
$$

for every $\boldsymbol{\eta} \in \mathscr{D}^{\mathbf{M}, N}\left(U^{\prime}\right)$.

Hence

$$
\mathbb{F}(D) \psi=\mathbb{F}(D) \phi
$$

as vector-valued ultradistributions on $U^{\prime 0}$.

Since the condition of the theorem implies that $\mathbb{F}(D)^{-1}$ is a matrix of local pseudo-differential operators, Eq. (3.15) is equivalent to $\psi=\phi$, as vector-valued ultra-distributions on $U^{\prime 0}$. But $\phi$ has support in $\bar{U}=U \cup \partial U$ and $\phi$ is the zero vector-valued ultradistribution on $U^{\prime 0}$.

Hence 
and, consequently, $\psi \in H(\partial D)$. This now implies that

$$
\mathbf{h}=\mathbb{F}^{*}(D) \psi=P_{U^{\prime}} \mathbf{f}
$$

belongs to $\mathscr{H}(\partial D)$, for every $\mathbf{f} \in \mathscr{H}(\bar{U})$.

This concludes the proof.

\section{Remark}

(i) The foregoing Theorem extends the work of [9] and [10] which only consider scalar ordinary Gaussian stochastic fields.

(ii) The Markovian fields characterized in Theorem (3.3) form a substantially wide class.

(iii) The conditions of Theorem (3.3) imply that $H^{0}(\Omega)$ is Markovian if and only if the Fourier-Laplace transform of the entries $\mathbb{P}_{i j}, i, j=1,2, \ldots, N$ of the matrix $\mathbb{P}$ are multipliers for the Fourier-Laplace image of $\mathscr{D}^{\mathbf{M}}(K)$ for every $K \in \operatorname{Comp}\left(\mathbb{R}^{d}\right)$. In Theorem (1.18), we supplied a characterization of the multipliers for the Fourier-Laplace image of $\mathscr{D}^{\mathbf{M}}(K), K \in \operatorname{Comp}\left(\mathbb{R}^{d}\right)$. For the purpose of applications (or for the sake of furnishing examples of multipliers) the following result which characterizes the set of entire functions which are multipliers for the Fourier-Laplace image of each of the spaces $\mathscr{D}^{\mathbf{M}}(K), K \in \operatorname{Comp}\left(\mathbb{R}^{d}\right)$ is evidently useful :

An entire function $\zeta \rightarrow E(\zeta), \zeta \in \mathbb{C}$, of one complex variable is a multiplier for the Fourier-Laplace image of $\mathscr{D}^{\mathbf{M}}(K), K \in \operatorname{Comp}\left(\mathbb{R}^{d}\right)$ if and only if

(a) it has Hadamard's factorization:

$$
E(\zeta)=\zeta^{n_{0}} \prod_{j=1}^{\infty}\left(1-\frac{\zeta}{c_{j}}\right), \quad n_{0}=a \text { positive integer }
$$

and

(b) for any $b>0$ there is $c>0$ such that the function

$$
\varrho \rightarrow N(\varrho)=\int_{0}^{\varrho} d \lambda \frac{n(\lambda)-n_{0}}{\lambda}
$$

satisfies the estimate

$$
N(\varrho) \leqq M(b \varrho)+\log c, \quad \varrho \in(0, \infty) .
$$

where $n(\lambda)$ is the number of $c_{j}$ such that $\left|c_{j}\right| \leqq \lambda$, and $M$ is as usual defined by (1.2)* and satisfies (1.2)**.

The result just quoted features as Proposition 7 of [15]. Using this result, one may now readily construct numerous examples of the functions $p \mapsto \tilde{\mathbb{P}}_{i j}(p), p \in \mathbb{R}^{d}$, $i, j=1,2, \ldots, N$, occurring in Theorem (3.3), which are the restrictions to $\mathbb{R}^{d}$ of multipliers for the Fourier-Laplace image of each of the spaces $\mathscr{D}^{\mathbf{M}}(K)$, $K \in \operatorname{Comp}\left(\mathbb{R}^{d}\right)$.

\section{References}

1. Levy, P.: Processus stochastique et mouvement Brownien. Paris: Gauthier-Villars 1948

2. Levy, P.: A special problem of Brownian motion, and a general theory of Gaussian random function. Proc. 3rd Berk. Symp. Math. Stat. Prob. 2, 133-175 (1956) 
3. Mckean, H.P., jr.: Brownian motion with a several dimensional time. Theory Probab. Its Appl. 8, 335-354 (1963)

4. Molchan, G.M.: On some problems concerning Brownian motion in Levy's sense. Theory Probab. Its Appl. 12, 682-690 (1967)

5. Pitt, L.D.: A Markov property for Gaussian processes with a multidimensional parameter. Arch. Ration. Mech. Anal. 43, 367-391 (1971)

6. Aronszajn, N.: Theory of reproducing kernels. Trans. Am. Math. Soc. 68, 337-404 (1950)

7. Peetre, J.: Rectification a l'article «Une caracterisation abstraite des operateurs differentiels». Math. Scand. 8, 116-120 (1960)

8. Lions, J.L., Magenes, E.: Non-homogeneous boundary value problems and applications, Vol. 1. Berlin, Heidelberg, New York: Springer 1972

9. Kotani, S.: On a Markov property for stationary Gaussian processes with a multidimensional parameter. In: Proc. of the Second Japan - USSR Symposium on probability theory. Lecture notes in mathematics, Vol. 330, pp. 239-250. Berlin, Heidelberg, New York: Springer 1973

10. Kotani, S., Okabe, Y.: On a Markovian property of stationary Gaussian processes with multidimensional parameter. In: Hyperfunctions and pseudo-differential equations. Lecture notes in mathematics, Vol. 287, pp. 153-169. Berlin, Heidelberg, New York: Springer 1973

11. Nelson, E. : Construction of quantum fields from Markoff fields. J. Funct. Anal. 12, 97-112 (1973)

12. Nelson, E.: The free Markoff field. J. Funct. Anal. 12, 211-227 (1973)

13. Simon, B.: The $P(\phi)_{2}$ Euclidean (quantum) field theory. In : Princeton series in physics. Princeton: Princeton University Press 1974

14. Velo, G., Wightman, A.: Constructive quantum field theory. Lecture notes in physics, Vol. 25. Berlin, Heidelberg, New York: Springer 1973

15. Komatsu, H.: Ultradistributions and hyperfunctions. Lecture notes in mathematics, Vol. 287, pp. 164-179. Berlin, Heidelberg, New York: Springer 1973

16. Roumieu, C.: Sur quelques extensions de la notion de distributions. Ann. Sci. Ec. Norm. Sup. 77, 47-121 (1960)

17. Roumieu, C.: Ultradistributions definies sur $\mathbb{R}^{n}$ et sur certaines classes de varieté's differentiables. J. Analyse Math. 10, 153-192 (1963)

18. Beurling, A.: Quasi-analyticity and general distributions. Lectures 4 and 5, Summer Institute, Stanford (1961)

19. Lions, J.C., Magenes, E.: Non-homogeneous boundary value problems and applications, Vol. III. Berlin, Heidelberg, New York: Springer 1973

20. Cristescu, R., Marinescu, G.: Applications of the theory of distributions. London, New York: Wiley 1973

21. Gel'fand, I.M., Vilenkin, N.Ya.: Generalized functions, Vol. 4. Applications of harmonic analysis. London, New York: Academic Press 1964

Communicated by H. Araki

Received June 12, 1979; in revised form November 15, 1979 
